\title{
Efficacy and Safety of Phospholipid Nanoemulsion-Based Ocular Lubricant for the Management of Various Subtypes of Dry Eye Disease: A Phase IV, Multicenter Trial
}

This article was published in the following Dove Press journal: Clinical Ophthalmology

\author{
Elizabeth Yeu' \\ Steven Silverstein $\mathbb{1 D}^{2}$ \\ Michel Guillon (iD ${ }^{3}$ \\ Marc-Matthias Schulze (DiD ${ }^{4}$ \\ David Galarreta (1D ${ }^{5}$ \\ Sruthi Srinivasan ${ }^{6}$ \\ Venkiteshwar Manoj ${ }^{6}$ \\ 'Virginia Eye Consultants, Norfolk, VA \\ 23502, USA; ${ }^{2}$ Silverstein Eye Centers, \\ Kansas City, MO 64I33, USA; ${ }^{3}$ Ocular \\ Technology Group International, London \\ SWIE 6AU, UK; ${ }^{4}$ Centre for Ocular \\ Research \& Education (CORE), School of \\ Optometry and Vision Science, \\ University of Waterloo, Waterloo, \\ Ontario NL2 3GI, Canada; ${ }^{5}$ Hospital \\ Clinico Universitario de Valladolid, \\ Valladolid Castillay Leon 470II, Spain; \\ ${ }^{6}$ Alcon Research, LLC, Johns Creek, GA \\ 30097, USA
}

Purpose: To evaluate the clinical efficacy and safety of propylene glycol/hydroxypropylguar (PG-HPG)-based nanoemulsion (Systane ${ }^{\circledR}$ Complete) lubricant eye drops in participants with dry eye disease (DED).

Participants and Methods: In this phase IV, open-label, single-arm, interventional, multicenter study, adult participants with DED - subtyped into aqueous deficient, evaporative, and mixed dry eye - were instructed to instill one drop of PG-HPG in each eye twice a day for 28 days. Endpoints included change from baseline in tear film break-up time (TFBUT) (primary) and ocular discomfort visual analog scale (VAS) score at Day 14 and TFBUT at Day 28 (secondary). Safety was assessed throughout the study. Data were analyzed for overall patient cohort and by DED subtypes.

Results: A total of 134 participants received treatment (mean age: 56.6 years; female: 75.4\%). At Day 14, the mean (standard deviation [SD]) TFBUT increased from 2.6 (1.00) seconds at baseline to 4.2 (2.92) seconds (mean change: 1.5 [2.80] seconds); median change was 0.8 seconds $(95 \%$ CI: $0.52,1.19)$ for the overall cohort; the change was maintained at Day 28 (mean change: 1.4 (2.80) seconds, median change: 0.8 seconds (95\% CI: $0.49,1.17$ ). Subgroup analysis showed a mean change (SD) from baseline in TFBUT of 1.1 (2.41) seconds for aqueous deficient, 2.4 (3.17) seconds for evaporative, and 1.2 (2.63) seconds for mixed dry eye at Day 14, respectively. The ocular discomfort VAS score improved (decreased) from baseline at Day 14 (mean [SD] change: -17.3 [24.80]) for the overall cohort, -22.0 [21.73] for aqueous deficient, -17.6 [24.17] for evaporative and -13.1 [27.49] for mixed dry eye subgroups). Adverse events (AEs) were reported in 9 (6.7\%) participants. No serious AEs were reported during the study.

Conclusion: Our study findings demonstrated that PG-HPG-based nanoemulsion lubricant eye drops were effective and well tolerated in participants with DED and all of its subtypes. Keywords: aqueous deficient dry eye, dry eye disease, evaporative dry eye, propylene glycol/hydroxypropyl guar-nanoemulsion ocular lubricant, mixed dry eye

\section{Introduction}

Dry eye is a multifactorial disease of the ocular surface, characterized by loss of homeostasis of the tear film and accompanied by ocular discomfort and disturbed vision, thereby impacting quality of life. ${ }^{1,2}$ As a leading reason that patients seek professional eye care, it poses a significant economic burden. ${ }^{3}$

Artificial tear products or ocular lubricants that attempt to replace and/or supplement the deficient natural tear film are the initial mainstay treatment option in the management
Correspondence: Elizabeth Yeu Virginia Eye Consultants, Norfolk, VA 23502, USA

Tel + I 757-622-2200

Fax + I 757-96I-00II

Email eyeu@vec2020.com 
of dry eye disease (DED). ${ }^{4}$ Most of the available artificial tears currently on the market target either aqueous deficient or evaporative DED. Selection of an appropriate artificial tear to address the needs of a patient may be challenging for general eye care practitioners or pharmacists who may not be capable of performing an eye examination utilizing the appropriate series of tests to diagnose the exact type of DED. $^{5}$ Furthermore, $30-70 \%$ of DED patients may suffer from symptoms of both aqueous deficient and evaporative type of DED (mixed type) and require treatment for both dry eye types for maximum relief. ${ }^{4}$ Thus, there is a need for a tear film ocular lubricant that can be used for managing all types of DED. Further, some of the significant challenges with ocular lubricants are the low retention time of active ingredients and blurred vision following instillation. ${ }^{4,6}$ In addition, a lipidbased ocular lubricant that can optimize the coverage area of lipids delivered to the ocular surface is an unmet need of patients with evaporative and mixed DED.

Systane $^{\circledR} \quad$ Complete lubricant eye drops (Alcon Laboratories, Inc. Fort Worth, TX, USA) is an innovative propylene glycol-hydroxypropyl-guar (PG-HPG) nanoemulsion, designed to replenish deficiencies in both the lipid and aqueous layers of the tear film. ${ }^{7}$ PG-HPG contains propylene glycol as an active demulcent, phospholipid nanoparticles to improve the coverage area of lipids delivered to the tear film and make the emulsion formulation less opaque (ie, translucent), and a higher amount of HPG to enhance the retention of lubricant on the ocular surface.
In a preclinical study, PG-HPG has been shown to provide effective moisture retention, protection, and improved cell barrier function and lubricity of corneal epithelium, which are required for management of all types of DED. ${ }^{8}$ However, the effect of this PG-HPG nanoemulsion-based eye drop in patients with all types of dry eye needed exploration.

The objective of this study was to evaluate the clinical effectiveness and safety of PG-HPG in adult study participants with all subtypes of DED.

\section{Materials and Methods Study Design}

Between July 2018 and May 2019, participants with all subtypes of DED (aqueous deficient, evaporative, and mixed) were enrolled at six centers across North America and Europe in this phase IV, 28-day, open-label, single-arm, interventional study (NCT03492541). The study consisted of two phases: the screening phase (Days -7 to 0 ), which assessed the study participants' eligibility, followed by the treatment phase (Days 1 to 28; Figure 1). Eligible study participants received the first dose of PG-HPG on Day 1 and were required to self-administer twice-daily dosing for 28 days with scheduled visits on Day 14 and 28; additional doses could be administered as needed if the study participants experienced dry eye symptoms.

Written informed consent was obtained from each participant before entry into the study. The study was approved by

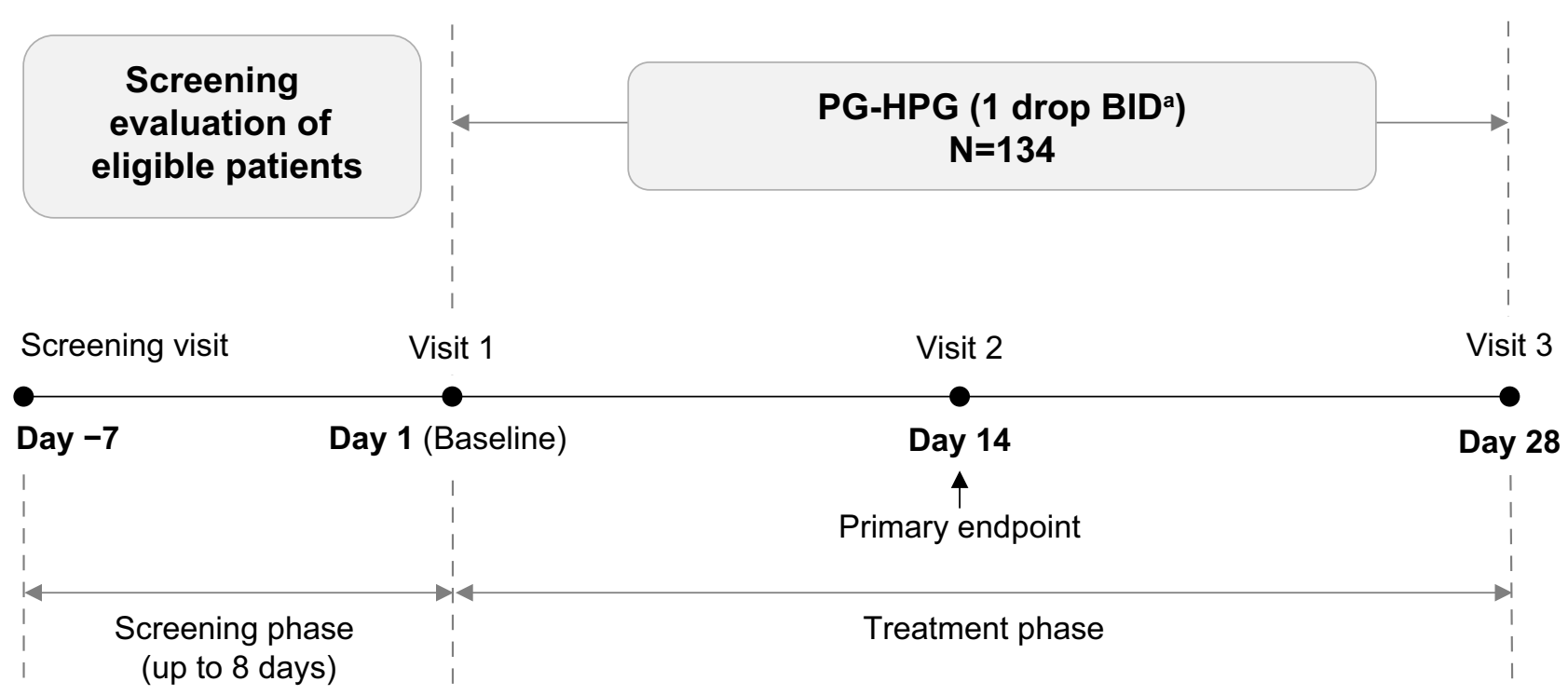

Figure I Study design. ${ }^{a}$ One drop was instilled twice-daily (morning and evening) in both eyes of eligible participants; additional doses were allowed in-between the two dosing intervals.

Abbreviations: BID, two times daily; PG-HPG, propylene glycol/hydroxypropyl guar-based lubricant eye drops. 
the independent ethics committee or institutional review board at each participating center and was conducted in accordance with the Declaration of Helsinki, the International Conference on Harmonization, and Good Clinical Practice guidelines (Appendix 1).

\section{Eligibility}

Participants aged $\geq 18$ years, having a tear film break-up time (TFBUT) of $\leq 5$ seconds in at least one eye, and best corrected visual acuity of $\geq 20 / 80$ (or $\geq 55$ letters score or $\geq 0.6$ early treatment diabetic retinopathy study log of the minimum angle of resolution value) in both eyes at screening were eligible for inclusion. In addition to the above, participants who met at least one of the three criteria below in at least one eye at screening (randomization stratified based on the criteria below) were also included: (a) unanesthetized Schirmer I test score of $\leq 9 \mathrm{~mm}$ for a five-minute test duration (aqueous deficient dry eye stratum); (b) meibum quality score of $\geq 1$ (on a 0 to 3 scale) or meibum expressibility score of $>1$ (on a 0 to 3 scale) in either eyelid (evaporative dry eye stratum); and (c) met both entry criteria (a) and (b) (mixed dry eye stratum). (Information on meibum quality and expressibility scores is available in Appendix 2). Participants were required to discontinue the use of any other artificial tear supplements and use only the study product for the entire study duration. Key exclusion criteria were (a) history of hypersensitivity to the study drug or any of its excipients; (b) use of any topical ocular medication preserved with benzalkonium chloride or other products known to be toxic to the tear film lipid layer, or lid hygiene therapy or punctal plug insertion within 1 month prior to the screening visit; (c) contact lens use within a week prior to screening visit; (d) initiation of any topical ocular medication (with the exception of artificial tears/gels/lubricants) $\leq 2$ weeks prior to the screening visit. Artificial tears/lubricants were prohibited from the beginning of the baseline visit until the patient was discontinued from the study.

\section{Objectives and Endpoints}

The primary objective was to evaluate improvement in tear film stability (ie, break-up) post-treatment with PG-HPG based on the change from baseline in TFBUT at Day 14. The secondary objectives of improvement of tear film stability, ocular discomfort, and improvement in corneal staining score with PG-HPG were assessed based on change from baseline in TFBUT at Day 28, in ocular discomfort Visual Analog Scale (VAS) score at Day 14, and in cornea staining score at Day 28, respectively. Data on adverse events (AEs) were collected throughout the study.

\section{Subgroup Analyses}

Subgroup analyses by dry eye subtypes (aqueous deficient, evaporative, or mixed) were performed on the primary and secondary endpoints.

\section{Assessments}

Assessments during each visit were conducted at the same time of day within \pm 1 hour of the start time of Visit 1 (baseline). TFBUT was measured 30 minutes following administration of study medication at screening, and Days 14 and 28 using an ocular staining dye, sodium fluorescein $(\mathrm{NaFl})$. Two microliters of $\mathrm{NaFl}$ solution were instilled in the right eye, immediately followed by three consecutive TFBUT measurements. The same procedure was followed for the left eye. The time from the last blink until one or more black (dry) spots appeared in the pre-corneal tear film was measured.

Ocular discomfort was evaluated using patient-reported outcome (PRO) assessments using VAS at baseline and Day 14 as per the study protocol. Study participants indicated their level of discomfort/level of severity on a 0-100 scale; a higher score indicated more frequent discomfort/ higher dry eye severity (Appendix 3).

Corneal staining score was assessed using NaFI, at screening, and Days 14 and 28 (or early exit) according to the procedure recommended by Bron et al. ${ }^{9}$

Details of the AEs and serious AEs (SAEs) were collected throughout the study.

\section{Statistical Analysis}

One hundred and twenty eligible patients were needed to estimate a $95 \%$ confidence interval (CI) with a precision of 0.38 and a standard deviation (SD) of 2.1 for the mean change from baseline at Day 14 in TFBUT (primary efficacy analysis). Considering a dropout rate of approximately $10 \%$, up to 134 participants were scheduled to be enrolled.

Descriptive statistics (mean, median, SD, minimum and maximum values) were presented for continuous variables (TFBUT, and ocular discomfort VAS score). In addition, a 95\% CI using Student's $t$-distribution was calculated for mean change from baseline at Day 14 in TFBUT. A 95\% CI for the median using the Hodges-Lehmann estimator was 
provided if the normality criteria were not met. Corneal staining was considered as a categorical measure.

Efficacy analysis was based on the full analysis set (FAS). Both FAS and safety analysis sets consisted of all participants who received at least one drop of PG-HPG lubricant eye drops.

The worse eye, defined as the eye with the lower TFBUT value at screening, of the participant was chosen for analysis. If both eyes had the same TFBUT value, then the right eye was considered the worse eye.

Data on AEs and SAEs were collected and analyzed for each eye of the participant.

All analyses were performed using SAS $^{\circledR}$ statistical software (Version 9.4).

\section{Results}

A total of 183 participants were evaluated for eligibility, of whom $134(73.2 \%)$ received treatment and $130(97 \%)$ completed the study. The reasons for study discontinuations were adverse event (2 [1.5\%]), withdrawal by the participant (1 [0.7\%]), and other $(1[0.7 \%])$ - this participant had a programmed cardiac procedure and was not available for study visits.

\section{Demographic and Baseline}

\section{Characteristics}

The mean (SD) age of participants was 56.6 (14.78) years and the majority of the participants were female $(75.4 \%)$. The numbers of participants for each of the dry eye subtypes (aqueous deficient, evaporative, and mixed) were similar (Table 1).

\section{Primary Outcome}

The mean TFBUT increased from 2.6 seconds at baseline to 4.2 seconds at Day 14 [mean (SD) change: 1.5 (2.80) seconds; the median change was 0.8 seconds $(95 \% \mathrm{CI}$ : $0.52,1.19)$ ] for the overall cohort. The results were consistent across the dry eye subgroups (Figure 2).

\section{Secondary Outcomes}

An increase in TFBUT at Day 14 was maintained through Day 28. In the overall cohort, the mean (SD) change from baseline in TFBUT at Day 28 was 1.4 (2.80) seconds and the median change was 0.8 seconds (95\% CI: $0.49,1.17)$. The results were comparable in the mixed-dry eye subgroup. The evaporative dry eye subgroup showed the largest change in mean (SD) TFBUT (2.5 [3.94] seconds). At
Table I Demographic and Baseline Characteristics (Full Analysis Set)

\begin{tabular}{|l|l|}
\hline Characteristics & $\begin{array}{l}\text { PG-HPG } \\
\text { N=134 }\end{array}$ \\
\hline Mean (SD) age, years & $56.6(14.8)$ \\
\hline $\begin{array}{l}\text { Age category, } \mathrm{n}(\%) \\
<65 \text { years }\end{array}$ & $88(65.7)$ \\
$\geq 65$ years & $46(34.3)$ \\
Gender, female, $\mathrm{n}(\%)$ & $10 \mathrm{I}(75.4)$ \\
\hline Race, $\mathrm{n}(\%)$ & \\
White & $100(74.6)$ \\
Black or African American & $23(17.2)$ \\
Asian & $6(4.5)$ \\
Other & $4(3.0)$ \\
Multiracial & $\mathrm{I}(0.7)$ \\
\hline Dry eye subtype - $\mathrm{n}$ (\%) & \\
Aqueous deficient & $4 \mathrm{I}(30.6)$ \\
Evaporative & $44(32.8)$ \\
Mixed & $49(36.6)$ \\
Mean (SD) ocular discomfort VAS score & $45.9(24.33)$ \\
Mean (SD) TFBUT, seconds & $2.6(1.0 \mathrm{I})$ \\
\hline
\end{tabular}

Notes: Ocular discomfort VAS score $=$ square root (VAS frequency score $\times$ VAS severity score). All baseline characteristics were assessed before instillation. Percentages are based on the number of participants in the full analysis set. Full analysis set consisted of all participants who received at least one drop of PG-HPG lubricant eye drops.

Abbreviations: PG-HPG, propylene glycol-hydroxypropyl guar lubricant eye drops; SD, standard deviation; TFBUT, tear film break-up time; VAS, visual analog scale.

Day 28, the aqueous deficient subgroup showed a marginal improvement of 0.6 (1.47) seconds in mean TFBUT from baseline (median change 0.3 seconds, $95 \% \mathrm{CI}-0.07,0.88$, Figure 3).

The ocular discomfort VAS score improved (decreased) from baseline at Day 14 (mean [SD] change -17.3 [24.80]) in the overall cohort. The dry eye subgroups followed a similar trend (Figure 4).

The cornea staining scores showed an improvement at Day 28 from baseline in the overall cohort as well as the dry eye subgroups (Table 2). Severe corneal staining was not reported during the study.

\section{Safety Outcomes}

Overall, 19 treatment-emergent AEs were reported in $9(6.7 \%)$ participants. Ocular AEs were reported in $6(4.5 \%)$ participants and are summarized in Table 3. One AE of blurred vision was reported; it was mild, transient, and was not considered to be related to the product per the investigator. Non-ocular AEs were 


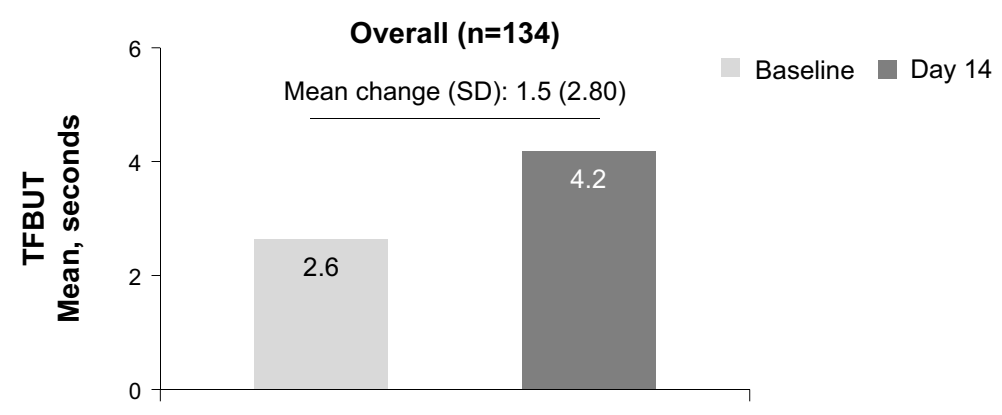

Aqueous deficient ( $n=41)$

Evaporative $(\mathrm{n}=44)$

Mixed ( $n=49)$

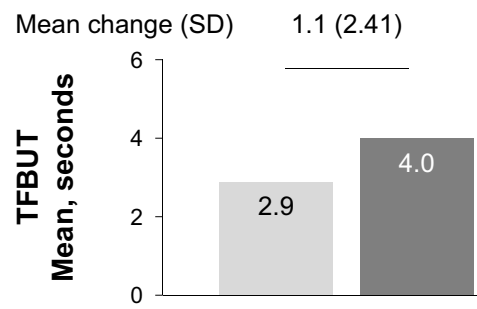

$2.4(3.17)$

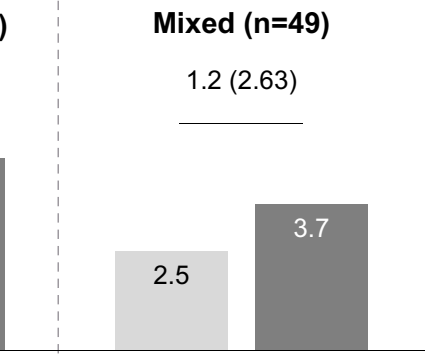

Figure 2 Change from baseline in TFBUT up to Day 14 in the overall cohort and dry eye subtypes (full analysis set). The $95 \% \mathrm{Cl}$ was calculated using Hodges-Lehmann estimator. Full analysis set consisted of all participants who received at least one drop of PG-HPG lubricant eye drops.

Abbreviations: $\mathrm{Cl}$, confidence interval; PG-HPG, propylene glycol-hydroxypropyl guar lubricant eye drops; TFBUT, tear film break-up time.

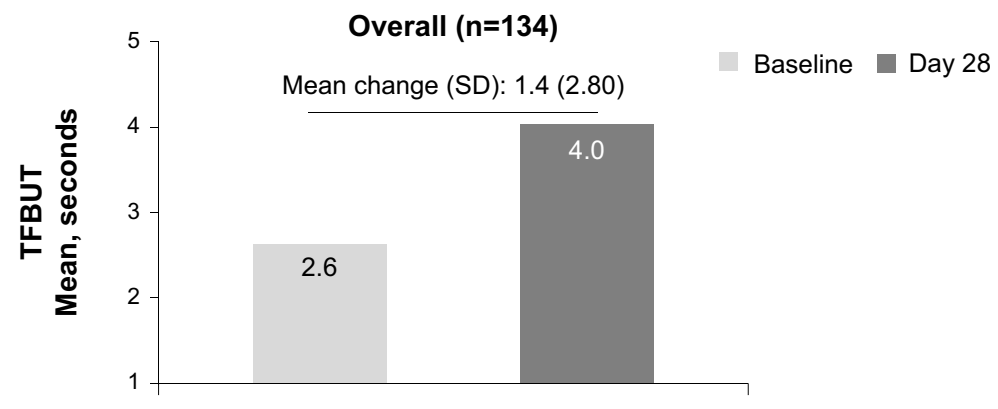

Aqueous deficient $(n=41)$

Evaporative $(n=44)$

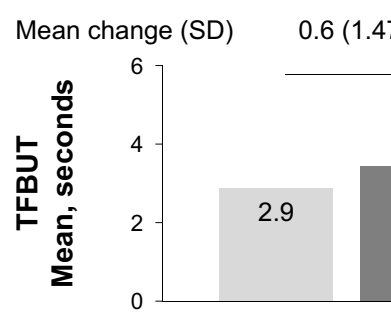

Mixed $(n=49)$

$1.1(2.08)$
2.5 (3.94)

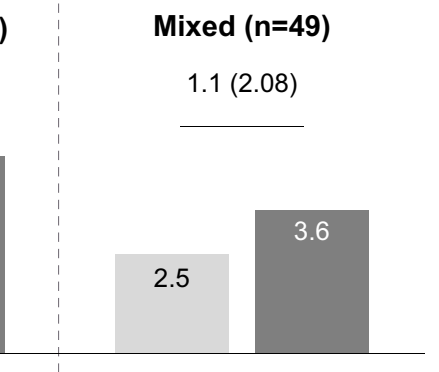

Figure 3 Change from baseline in TFBUT up to Day 28 in the overall cohort and dry eye subtypes (full analysis set). The $95 \% \mathrm{Cl}$ was calculated using Hodges-Lehmann estimator. Full analysis set consisted of all participants who received at least one drop of PG-HPG lubricant eye drops. Baseline was defined as the last available, non-missing, scheduled or unscheduled value collected prior to exposure to study treatment.

Abbreviations: $\mathrm{Cl}$, confidence interval; PG-HPG, propylene glycol-hydroxypropyl guar lubricant eye drops; TFBUT, tear film break-up time.

reported in $8(6 \%)$ participants (headache in 3 [2.2\%], nasopharyngitis in 2 [1.5\%], influenza-like illness, arthritis and musculoskeletal chest pain in $1(0.7 \%)$ patient each). None of the ocular and non-ocular AEs reported were severe. Two participants discontinued the treatment owing to ocular AEs (eye pruritus and viral 

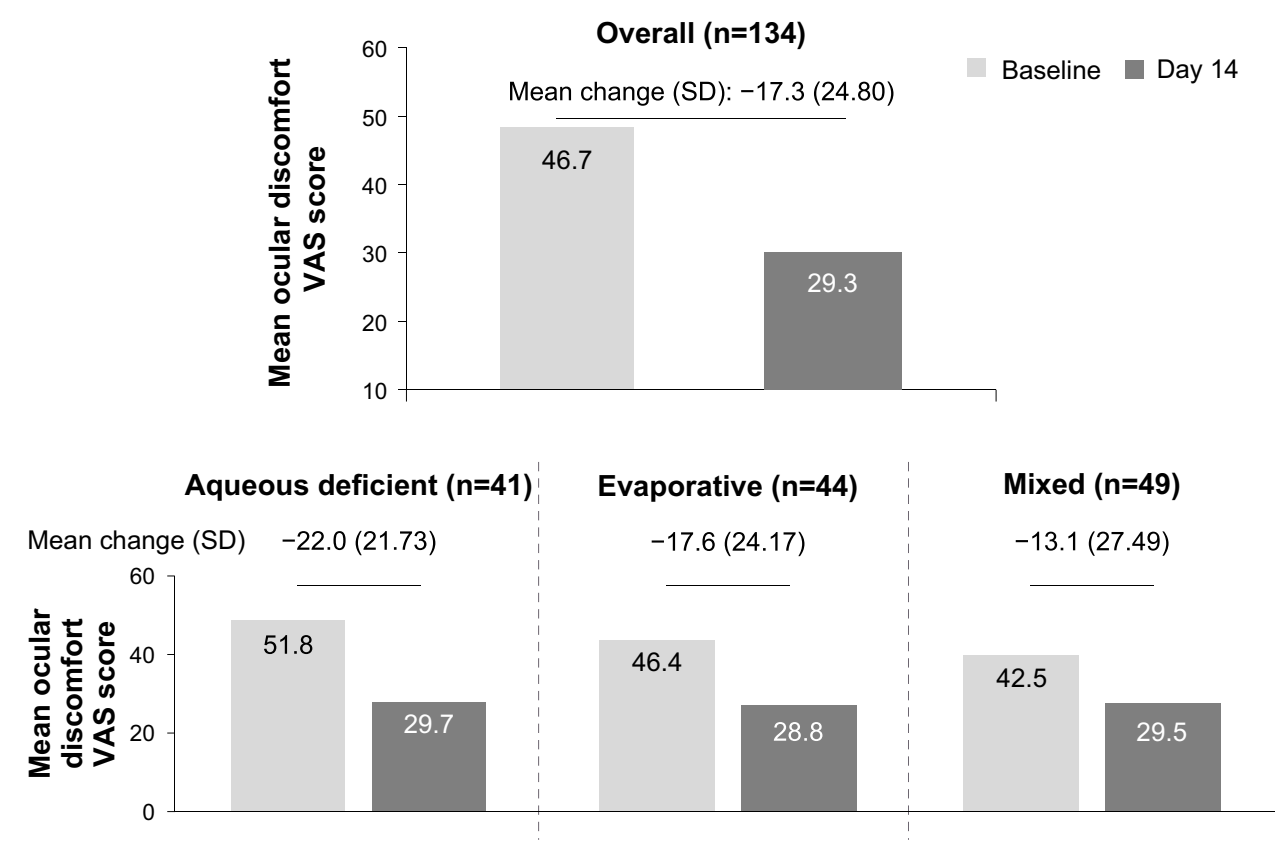

Figure 4 Change from baseline in ocular discomfort VAS score at Day 14 in the overall cohort and dry eye subtypes (full analysis set). Full analysis set consisted of all participants who received at least one drop of PG-HPG lubricant eye drops. Baseline was defined as the last available, non-missing, scheduled or unscheduled value collected prior to exposure to study treatment.

Abbreviations: PG-HPG, propylene glycol-hydroxypropyl guar lubricant eye drops; SD, standard deviation; VAS, visual analog scale.

conjunctivitis; $1[0.7 \%]$ patient each). SAEs were not reported during the study.

\section{Discussion}

The results of this clinical study demonstrated that treatment with PG-HPG nanoemulsion-based lubricant eye drops enhanced the tear film stability in participants with DED, thereby meeting its primary objective. This improvement was sustained through Day 28. In addition, PG-HPG also improved the signs and symptoms of participants with DED, and was considered to be well tolerated throughout the study.

PG-HPG nanoemulsion-based lubricant eye drop is the first Systane ${ }^{\circledR}$ product that combines the benefits of HPG and nanoparticle technology (reduced lipid droplet size $<100 \mathrm{~nm}$ ) to replenish both the aqueous and lipid deficiencies of the tear film, potentially targeting all subtypes of dry eye. Following application of PG/HPGnanoemulsion, HPG and borate form a cross-linked matrix which acts as a protective viscoelastic barrier on the ocular surface epithelium. This barrier improves the retention of the demulcent (PG) on the aqueous/mucin layer and moisture content of the deficient aqueous tear film, and also acts as a depot for slow release of lipid into the tear film, to supplement and stabilize any gaps due to lipid insufficiency. The anionic phospholipids in
PG-HPG can be beneficial for the proper structuring of the polar lipid layer and maintaining tear film stability by acting as an effective interface between the outer nonpolar lipids and the underlying aqueous phase as evident from previous studies. ${ }^{8,10}$ Thus, PG-HPG-nanoemulsion restores the complete tear film structure to prevent exacerbations of DED and maintain a healthy ocular surface. $^{7}$

The beneficial effect of HPG technology facilitating the replenishment of the deficient tear film is well reported. ${ }^{11-14}$ Systane ${ }^{\circledR}$ Balance (Alcon Laboratories, Inc. Fort Worth, TX, USA), which is an oil-based HPG emulsion product, helps to replenish the lipid layer in a lipid-deficient tear film. ${ }^{11}$ Systane $^{\circledR}$ and Systane ${ }^{\circledR}$ Ultra lubricant eye drops (Alcon Laboratories, Inc. Fort Worth, TX, USA) utilize the HPG technology and provide long-lasting relief from symptoms of dryness of the eye and sustained comfort. ${ }^{12-14}$

PG-HPG utilizes the same active demulcent as Systane ${ }^{\mathbb{B}}$ Balance, ie, $\mathrm{PG}$, but with increased concentration of HPG which allows for better crosslinking with borate. PG-HPG has been uniquely processed compared to Systane ${ }^{\circledR}$ Balance. The smaller nano-sized lipid droplets in PG-HPG optimize the lipid surface coverage and make the emulsion formulation less opaque compared with other Systane ${ }^{\circledR}$ lubricant eye drops, reducing blurred vision upon instillation of ocular lubricants. ${ }^{4,13,15}$ 
Table 2 Change in Cornea Staining Score from Baseline to Day 28 (Full Analysis Set)

\begin{tabular}{|c|c|c|c|c|c|c|c|}
\hline \multirow{2}{*}{\multicolumn{2}{|c|}{ Baseline Score, n }} & \multicolumn{6}{|c|}{$\begin{array}{l}\text { PG-HPG }(\mathrm{N}=134) \\
\text { Post-Baseline (Day 28) Scores }\end{array}$} \\
\hline & & \multirow[t]{2}{*}{$\begin{array}{l}\text { Absent } \\
\text { n (\%) }\end{array}$} & \multirow[t]{2}{*}{$\begin{array}{l}\text { Minimal } \\
\text { n (\%) }\end{array}$} & \multirow[t]{2}{*}{$\begin{array}{l}\text { Mild } \\
\text { n (\%) }\end{array}$} & \multirow[t]{2}{*}{$\begin{array}{l}\text { Moderate } \\
\text { n (\%) }\end{array}$} & \multirow[t]{2}{*}{$\begin{array}{l}\text { Marked } \\
\text { n (\%) }\end{array}$} & \multirow[t]{2}{*}{$\begin{array}{l}\text { Missing } \\
\text { n (\%) }\end{array}$} \\
\hline Full cohort & & & & & & & \\
\hline Absent & 48 & $36(75.0)$ & $9(18.8)$ & $2(4.2)$ & 0 & 0 & $I(2.1)$ \\
\hline Minimal & 43 & $14(32.6)$ & $24(55.8)$ & $4(9.3)$ & 0 & 0 & I (2.3) \\
\hline Mild & 34 & $5(14.7)$ & $12(35.3)$ & $14(4 \mid .2)$ & I (2.9) & I (2.9) & I (2.9) \\
\hline Moderate & 9 & $2(22.2)$ & $2(22.2)$ & $\mathrm{I}(\mathrm{II} . \mathrm{I})$ & $3(33.3)$ & 0 & I (II.I) \\
\hline Marked & 0 & 0 & 0 & 0 & 0 & 0 & 0 \\
\hline Total & 134 & $57(42.5)$ & $47(35.1)$ & $21(15.7)$ & $4(3.0)$ & I (0.7) & $4(3.0)$ \\
\hline \multicolumn{8}{|c|}{ Aqueous deficient ${ }^{a}$} \\
\hline Absent & 10 & $6(60)$ & $3(30.0)$ & $\mathrm{I}(10.0)$ & 0 & 0 & 0 \\
\hline Minimal & 17 & $7(4 \mid .2)$ & $9(52.9)$ & I (5.9) & 0 & 0 & 0 \\
\hline Mild & 12 & $2(16.7)$ & $5(41.7)$ & $4(33.3)$ & 0 & I (8.3) & 0 \\
\hline Moderate & 2 & 0 & I (50.0) & I (50.0) & 0 & 0 & 0 \\
\hline Total & 41 & $15(36.6)$ & $18(43.9)$ & $7(17.1)$ & 0 & I (2.4) & 0 \\
\hline \multicolumn{8}{|c|}{ Evaporative $^{b}$} \\
\hline Absent & 23 & $18(78.3)$ & $4(17.4)$ & I (4.3) & 0 & 0 & 0 \\
\hline Minimal & 12 & $5(4 \mid .7)$ & $6(50.0)$ & $\mathrm{I}(8.3)$ & 0 & 0 & 0 \\
\hline Mild & 8 & I (12.5) & I (I2.5) & $5(62.5)$ & $I(12.5)$ & 0 & 0 \\
\hline Moderate & I & 0 & 0 & 0 & 0 & 0 & I $(100)$ \\
\hline Total & 44 & $24(54.5)$ & II (25.0) & 7 (I5.9) & I (2.3) & 0 & I (2.3) \\
\hline \multicolumn{8}{|l|}{ Mixed $^{b}$} \\
\hline Absent & 15 & $12(80.0)$ & $2(13.3)$ & 0 & 0 & 0 & I (6.7) \\
\hline Minimal & 14 & $2(14.3)$ & $9(64.3)$ & $2(14.3)$ & 0 & 0 & I (7.I) \\
\hline Mild & 14 & $2(14.3)$ & $6(42.9)$ & $5(35.7)$ & 0 & 0 & I (7.I) \\
\hline Moderate & 6 & $2(33.3)$ & I (16.7) & 0 & $3(50.0)$ & 0 & 0 \\
\hline Total & 49 & $18(36.7)$ & $18(36.7)$ & $7(14.3)$ & $3(6.1)$ & 0 & $3(6.1)$ \\
\hline
\end{tabular}

Notes: ${ }^{\text {a }}$ There were no participants with severe cornea staining score at baseline and Day 28 . ${ }^{\text {}}$ There were no participants with marked or severe cornea staining score at baseline and Day 28. Percentages are based on the number of participants with worse eye in the respective baseline score category in the full analysis set. Full analysis set consisted of all participants who received at least one drop of PG-HPG lubricant eye drops.

Abbreviations: PG-HPG, propylene glycol-hydroxypropyl guar lubricant eye drops; TFBUT, tear film break-up time.

Tear film stability, which is essential for ocular surface homeostasis and functioning, is disturbed in DED. ${ }^{16}$ The TFBUT, constituting the time interval elapsed between a complete blink and the appearance of the first break in the tear film, is a direct test of tear film stability and a valuable diagnostic aid in DED. ${ }^{17}$ In the present study, treatment with PG-HPG nanoemulsion elicited an increase of 1.5 seconds (59\% improvement) from baseline $(2.6$ seconds) in mean TFBUT at Day 14. A previous study has reported that a decrease in one second of TFBUT can have a major impact on ocular discomfort in $>70 \%$ of DED patients. $^{18}$ Thus, considering the low baseline value, the increase in TFBUT ( $>1$ second) observed with short-term use of PG-HPG nanoemulsion, though modest, is yet meaningful for DED patients. However, the variability observed in TFBUT outcomes may be attributed to factors (eg, residual tear volume, environmental conditions, etc.) that are known to influence TFBUT measurements. ${ }^{19}$ Earlier studies have reported that HPG in Systane ${ }^{\circledR}$ formulations interacts with the divalent ions and mucin in the natural tear film to form a gel-matrix, thus contributing to the tear film stability. ${ }^{12,13,20}$ The results of this study demonstrate the ability of PG-HPG to promote tear film stability in participants with DED, in agreement with earlier studies which showed an increased TFBUT with Systane ${ }^{\circledR}$ Balance in patients with evaporative DED and with Systane ${ }^{\circledR}$ lubricant eye drops in patients with DED. ${ }^{13,21}$

The symptoms of ocular surface disorder improved after PG-HPG instillation - the ocular discomfort VAS 
Table 3 Proportion (\%) of Participants Reporting Ocular Adverse Events (Safety Analysis Set)

\begin{tabular}{|l|l|}
\hline $\begin{array}{l}\text { System Organ Class } \\
\text { Preferred Terms }\end{array}$ & PG-HPG N=I34 \\
\hline Eye disorders & $5(3.7)$ \\
Halo vision & $\mathrm{I}(0.7)$ \\
Conjunctival hyperemia & $\mathrm{I}(0.7)$ \\
Eye pruritus & $\mathrm{I}(0.7)$ \\
Eyelid margin crusting & $\mathrm{I}(0.7)$ \\
Vision blurred & $\mathrm{I}(0.7)$ \\
Infections and infestations & $\mathrm{I}(0.7)$ \\
Conjunctivitis viral & $\mathrm{I}(0.7)$ \\
\hline
\end{tabular}

Notes: A patient with multiple AEs within a preferred term was counted only once for that preferred term. MedDRA Version 20.I was used for the reporting of AEs, Safety analysis set consisted of all participants who received at least one drop of PG-HPG lubricant eye drops.

Abbreviations: AE, adverse event; PG-HPG, propylene glycol-hydroxypropyl guar lubricant eye drops.

score decreased at Day 14, and there was a reduction in corneal staining score at Day 28 relative to baseline. The improvement in dry eye symptoms may be attributed to the increase in tear film stability and the beneficial effect of the HPG technology - longer retention of the demulcent, thus reducing the friction between lid and ocular surface. Lipid nanoparticles in this formulation are designed for better lipid coverage of the tear film, contributing to a reduction in the ocular discomfort of patients suffering from DED. These results indicate that PG-HPG nanoemulsion can provide symptomatic relief in patients with DED and are consistent with those reported with other Systane ${ }^{\circledR}$ eye drops. ${ }^{11,14,22}$

Findings in the subgroups based on the dry eye subtypes were comparable to that of the overall study cohort. The only exception was the change in TFBUT at Day 28 for the aqueous subgroup, which showed a nominal change from baseline based on $95 \%$ CI values $(-0.07$, 0.88). The effect on TFBUT was pronounced in the evaporative subgroup as compared to other subgroups, possibly because PG-HPG was formulated by processing of ingredients similar to that of Systane ${ }^{\circledR}$ Balance lubricant eye drops, which have a known efficacy in patients with evaporative DED. Previous studies have shown that anionic phospholipids can provide a stable interface between nonpolar lipids at the surface of the tear film and the aqueous layer, and polar lipids can enhance the lipid layer thickness. ${ }^{6,10}$ The reduced droplet size of nanoemulsion is expected to serve as a better delivery vehicle for lipids to the ocular surface to help optimize the evaporation rate of the tear film. However, studies involving a larger population are warranted to further ascertain the efficacy across all dry eye subtypes.

Selection of an appropriate ocular lubricant is influenced by the cause of DED (aqueous deficiency, lipid deficiency, or both) and is critical for the management of DED. ${ }^{4}$ Patients with evaporative DED require ocular lubricants, which reduce the rate of tear evaporation. In addition to treating the root cause, for patients with meibomian gland dysfunction, replenishment of the tear film with lipids is important. PG-HPG nanoemulsion treatment acts by replenishing the lipid layer and leads to an increase in tear film stability, potentially reducing the rate of tear evaporation in patients with evaporative dry eye. Patients with aqueous deficient DED have low tear volume and require treatments that can sufficiently hydrate the ocular surface. ${ }^{4}$ The lubricant and HPG technology in PG-HPG nanoemulsion reduce the ocular discomfort in these aqueous-deficient patients. If only one of these two broad categories of ocular surface disease (evaporative dry eye and aqueous deficient dry eye) is addressed therapeutically, patients may continue to experience symptoms and report dissatisfaction with the prescribed treatment. ${ }^{4}$ PG-HPG nanoemulsion-based lubricant eye drops can serve as a total tear replacement technology in all types of DED.

Treatment of participants with PG-HPG nanoemulsion was well tolerated. The proportion of patients with blurred vision upon instillation of PG-HPG was low in comparison with other Systane ${ }^{\circledR}$ eye drops. ${ }^{13,15}$ HPG-based ocular lubricants usually cause blurred vision. ${ }^{13,15}$ However, in this study the investigator did not consider blurred vision to be treatment-related. This may be attributed to the small nano-sized droplets in PG-HPG nanoemulsion, which make it less opaque. Neither SAEs nor severe AEs were reported, similar to that known for other Systane ${ }^{\circledR}$ eye drops. ${ }^{11,23,24}$

The strength of this study is that data were collected from six sites across different countries. The study is limited by the invasiveness of fluorescein TFBUT measurements typically used by clinicians. Further, this was a single-arm study; a comparison of PG-HPG with existing market products was not performed due to the unique combination of HPG and nanotechnology in this formulation. The effect of vehicle in PG-HPG nanoemulsion has been studied earlier in a preclinical study demonstrating better (32-40 times) hydration protection, lubricity, and polymer film break-up time (all $P<0.05$ ) as compared to vehicle (nanoemulsion without HPG) in human corneal 
epithelial cells. ${ }^{8}$ However, the inclusion of a comparator/ placebo arm can be considered in future clinical studies.

\section{Conclusions}

Our study findings showed that PG-HPG nanoemulsionbased lubricant eye drops improved the tear film stability, signs and symptoms of DED, and were well tolerated in participants with DED irrespective of its subtypes.

\section{Data Sharing Statement}

Due to the varying rights of individuals and contractual rights of parties involved, Alcon does not make a practice of sharing datasets.

\section{Acknowledgments}

The medical writing support and editorial assistance during the development of the manuscript were provided by Swati Bhandari, PhD (Novartis Healthcare Pvt. Ltd, Hyderabad, India). Alcon would like to thank the principal investigators, Ali Mearza, Joseph Tauber, and Lyndon Jones for their contributions to the study. This study is registered with ClinicalTrials.gov as NCT03492541.

\section{Funding}

The study was funded by Alcon Research LLC, Fort Worth, Texas, US.

\section{Disclosure}

Elizabeth Yeu: Consultant for Alcon, Allergan, Avedro, Avellino, Bausch + Lomb, BioTissue/Tissue Tech, Bruder, BVI, CorneaGen, EyePoint Pharm, ExpertOpinion.MD, Glaukos, iOptics, J\&J Vision, LENSAR, Kala Pharm., Merck, Mynosys, Novartis, Ocular Science, Ocular Therapeutix, OCuSOFT, Omeros Oyster Point Pharm, ScienceBased Health, Sun Pharm. TearLab Corp. Carl Zeiss. Investor in Avellino, CorneaGen, Melt, Modernizing Medicine, Mynosys, Ocular Science, Strathspey Crown. Research for Alcon, Allergan, TopCon, AcuFocus. Steven Silverstein: Consultant and Research-Alcon. Michel Guillon: Employee of Optometric Technology Group Ltd, consultant and financial support: Alcon. Marc Schulze: His research group (CORE) has received research support: Alcon, Allergan, Contamac, CooperVision, GL Chemtec, Inflamax Research, J\&J Vision, Menicon, Nature's Way, Novartis, PS Therapy, Shire, SightGlass and Visioneering. David Galarreta: Consultant to Alcon, Bausch \& Lomb, Novartis, Santen, Thea, VISUfarma. Research for Alcon, Allergan, Nicox, Novartis, Sifi. Sruthi Srinivasan and Venkiteshwar Manoj: Employees of
Alcon. The authors report no other conflicts of interest in this work.

\section{References}

1. Craig JP, Nichols KK, Akpek EK, et al. TFOS DEWS II definition and classification report. Ocul Surf. 2017;15(3):276-283. doi:10.1016/j.jtos.2017.05.008

2. Friedman NJ. Impact of dry eye disease and treatment on quality of life. Curr Opin Ophthalmol. 2010;21(4):310-316. doi:10.1097/ ICU.0b013e32833a8c15

3. Available from: https://eyewiki.aao.org/Dry_Eye_Syndrome. Assessed 28 Nov 2019.

4. Jones L, Downie LE, Korb D, et al. TFOS DEWS II management and therapy report. Ocul Surf. 2017;15(3):575-628. doi:10.1016/j. jtos.2017.05.006

5. Bilkhu PS, Wolffsohn JS, Tang GW, Naroo SA. Management of dry eye in UK pharmacies. Cont Lens Anterior Eye. 2014;37(5):382-387. doi:10.1016/j.clae.2014.06.002

6. Lallemand F, Daull P, Benita S, Buggage R, Garrigue JS. Successfully improving ocular drug delivery using the cationic nanoemulsion, novasorb. J Drug Deliv. 2012;2012:604204. doi: $10.1155 / 2012 / 604204$

7. Available from: https://systane.myalcon.com/eye-care/systane/pro ducts/systane-complete/. Accessed Nov 25, 2019.

8. Rangarajan $R$, Ketelson $H$. Preclinical evaluation of a new hydroxypropyl-guar phospholipid nanoemulsion-based artificial tear formulation in models of corneal epithelium. J Ocul Pharmacol Ther. 2019;35(1):32-37. doi:10.1089/jop.2018.0031

9. Bron AJ, Evans VE, Smith JA. Grading of corneal and conjunctival staining in the context of other dry eye tests. Cornea. 2003;22 (7):640-650. doi:10.1097/00003226-200310000-00008

10. Korb DR, Greiner JV, Glonek T. The effects of anionic and zwitterionic phospholipids on the tear film lipid layer. Adv Exp Med Biol. 2002;506(Pt A):495-499.

11. Aguilar AJ, Marquez MI, Albera PA, Tredicce JL, Berra A. Effects of systane $((\mathrm{R}))$ balance on noninvasive tear film break-up time in patients with lipid-deficient dry eye. Clin Ophthalmol. 2014;8:2365-2372. doi:10.2147/OPTH.S70623

12. Hartstein I, Khwarg S, Przydryga J. An open-label evaluation of HP-guar gellable lubricant eye drops for the improvement of dry eye signs and symptoms in a moderate dry eye adult population. Curr Med Res Opin. 2005;21(2):255-260. doi:10.1185/030079 $905 \times 26252$

13. Gifford P, Evans BJ, Morris J. A clinical evaluation of systane. Cont Lens Anterior Eye. 2006;29(1):31-40. doi:10.1016/j.clae.2005.12.003

14. Davitt WF, Bloomenstein M, Christensen M, Martin AE. Efficacy in patients with dry eye after treatment with a new lubricant eye drop formulation. J Ocul Pharmacol Ther. 2010;26(4):347-353. doi:10.1089/jop.2010.0025

15. Labetoulle M, Schmickler S, Galarreta D, et al. Efficacy and safety of dual-polymer hydroxypropyl guar- and hyaluronic acid-containing lubricant eyedrops for the management of dry-eye disease: a randomized double-masked clinical study. Clin Ophthalmol. 2018;12:2499-2508. doi:10.2147/OPTH.S177176

16. Willcox MDP, Argueso P, Georgiev GA, et al. TFOS DEWS II tear film report. Ocul Surf. 2017;15(3):366-403. doi:10.1016/j.jtos. 2017.03.006

17. Wolffsohn JS, Arita R, Chalmers R, et al. TFOS DEWS II diagnostic methodology report. Ocul Surf. 2017;15(3):539-574. doi:10.1016/j. jtos.2017.05.001

18. Nally L, Ousler GW, Abelson MB. Ocular discomfort and tear film break-up time in dry eye patients: a correlation. IOVS. 2000;41(4 (ARVO Abstract)):1436. 
19. McMonnies CW. Tear instability importance, mechanisms, validity and reliability of assessment. J Optom. 2018;11(4):203-210. doi:10.1016/j.optom.2017.11.004

20. Kading D. A two-week clinical evaluation of the safety of systane ultra in contact lens-wearing patients. Clin Ophthalmol. 2010;4:27-32. doi:10.2147/OPTH.S8079

21. Korb DR, Blackie C, Meadows D, Christensen MT, Tudor MR. Evaluation of extended tear stability by two emulsion based artificial tears. In: Proceedings of the 6th International Conference on the Tear Film and Ocular Surface: Basic Science and Clinical Relevance Tear Film and Ocular Surface Society meeting, Florence, Italy, September $22-25,2010$.
22. Christensen MT. Corneal staining reductions observed after treatment with systane lubricant eye drops. Adv Ther. 2008;25(11):1191-1199. doi:10.1007/s12325-008-0112-0

23. Asbell P, Vingrys AJ, Tan J, et al. Clinical outcomes of fixed versus as-needed use of artificial tears in dry eye disease: a 6-week, observer-masked phase 4 clinical trial. Invest Ophthalmol Vis Sci. 2018;59(6):2275-2280. doi:10.1167/iovs.17-23733

24. Fernandez KB, Epstein SP, Raynor GS, et al. Modulation of HLA-DR in dry eye patients following 30 days of treatment with a lubricant eyedrop solution. Clin Ophthalmol. 2015;9:1137-1145. doi:10.2147/ OPTH.S81355
Clinical Ophthalmology

\section{Publish your work in this journal}

Clinical Ophthalmology is an international, peer-reviewed journal covering all subspecialties within ophthalmology. Key topics include: Optometry; Visual science; Pharmacology and drug therapy in eye diseases; Basic Sciences; Primary and Secondary eye care; Patient Safety and Quality of Care Improvements. This journal is indexed on PubMed

Submit your manuscript here: https://www.dovepress.com/clinical-ophthalmology-journal
Dovepress

Central and CAS, and is the official journal of The Society of Clinical Ophthalmology (SCO). The manuscript management system is completely online and includes a very quick and fair peer-review system, which is all easy to use. Visit http://www.dovepress.com/ testimonials.php to read real quotes from published authors. 